\title{
Dem Fachkräftemangel das Handwerk legen
}

\section{Entwicklung und Evaluation eines Instruments zur bedürfnisorientierten Arbeitsanalyse und Strukturierung von Mitarbeitendengesprächen im Handwerk}

\author{
Christian Jaster ${ }^{1}(0)$ und Thomas Ellwart ${ }^{2}$ (i) \\ 1 Moldzio \& Partner - Institut für Personalauswahl, Ahrensburg \\ ${ }^{2}$ Abteilung Wirtschaftspsychologie, Universität Trier
}

\begin{abstract}
Zusammenfassung: Der Fachkräftemangel stellt besonders im Handwerk große Anforderungen an die Führungskräfte. Um Führungskräfte im Handwerk bei der mitarbeiterorientierten Führung zu unterstützen, wurde ein Instrument zur bedürfnisorientierten Arbeitsanalyse (MotivSORT) entwickelt und pilotiert. Mit MotivSORT befragen Führungskräfte die Mitarbeitenden zu 15 Arbeitsmerkmalen auf den Dimensionen (1) Wichtigkeit und (2) Ausprägung im Arbeitsalltag. Die Validität und Akzeptanz von MotivSORT wurde in zwei Studien mit Mitarbeitenden im Handwerk ( $\left.N_{1}=51 ; N_{2}=203\right)$ untersucht. Eine Überprüfung des formativen Messmodells zeigte Konvergenzvalidität und erwartungskonforme Zusammenhänge zwischen der Ausprägung bedürfnisbezogener Arbeitsmerkmale und Indikatoren der Mitarbeitendenbindung (z. B. Arbeitszufriedenheit, affektive Bindung, Kündigungsabsicht). Zudem zeigte sich eine hohe Akzeptanz von MotivSORT seitens der Mitarbeitenden auf den Dimensionen Nützlichkeit, Einfachheit der Anwendung, Arbeitsplatzrelevanz und Nutzungsabsicht. MotivSORT wird aktuell in der Betriebsberatung der Handwerkskammern Rheinland-Pfalz zur mitarbeiterorientierten Führung sowie Organisationsdiagnose implementiert. Perspektivisch wird die Anwendung auf weitere Branchen und organisational relevante Themen wie Personalauswahl und Veränderungsprozesse erweitert.
\end{abstract}

Schlüsselwörter: Personalentwicklung, Arbeitsgestaltung, Mitarbeiterorientierte Führung, Organisationsdiagnostik, Fachkräftemangel

Putting a Stop to Skilled Laborer Shortages. The Development and Evaluation of a Screening Tool for Need-Oriented Work Analysis and Structured Employee Interviews in the Craft Sector

Abstract: The shortage of skilled workers poses a significant challenge for managers in the craft sector. To support these managers in employee-oriented leadership, we developed and piloted a screening tool of need-oriented work design (MotivSORT), where employees rate 15 work characteristics on the dimensions of (1) personal need and (2) current supply. We tested the validity and acceptance of MotivSORT in two separate employee studies $\left(N_{1}=51 ; N_{2}=203\right)$. The formative model indicated a construct validity and high correlations between the satisfaction of work characteristics and employee commitment. Employees were highly acceptant of MotivSORT on the dimensions of usefulness, ease of use, job relevance, and intention to use. MotivSORT is currently being integrated into the consultancy portfolio of the Trade Chamber of Rhineland-Palatinate. Application of MotivSORT to new areas such as change management is discussed.

Keywords: Human Resource Development, Work Design, Employee-oriented Leadership, Organizational Diagnostics, Skilled Worker Shortage

\section{Ziele des Projekts}

\section{Strukturierte Mitarbeitendengespräche in Zeiten des Fachkräftemangels}

Fachkräftemangel stellt besondere Anforderungen an die Vorgesetzten. Gerade das Handwerk ist aufgrund seiner kleinbetrieblichen Struktur sowie einer schwachen Arbeitsmarktposition stark betroffen (Paulus, 2020). In die- sen Kleinbetrieben wird die Fachkräftesicherung durch eine weniger institutionalisierte Personalarbeit erschwert (Schirner et al., 2021). Die Folgen sind ein stetig wachsender Konkurrenzdruck und Abwanderung aus dem Handwerk in andere, meist finanzstärkere Wirtschaftsbereiche (Thomä, 2014). Dieser Abwanderung können Handwerksbetriebe begegnen, indem sie verstärkt auf nicht-monetäre Anreize wie Arbeitsplatzsicherheit, Betriebsklima oder den Einsatz moderne Technologien set- 


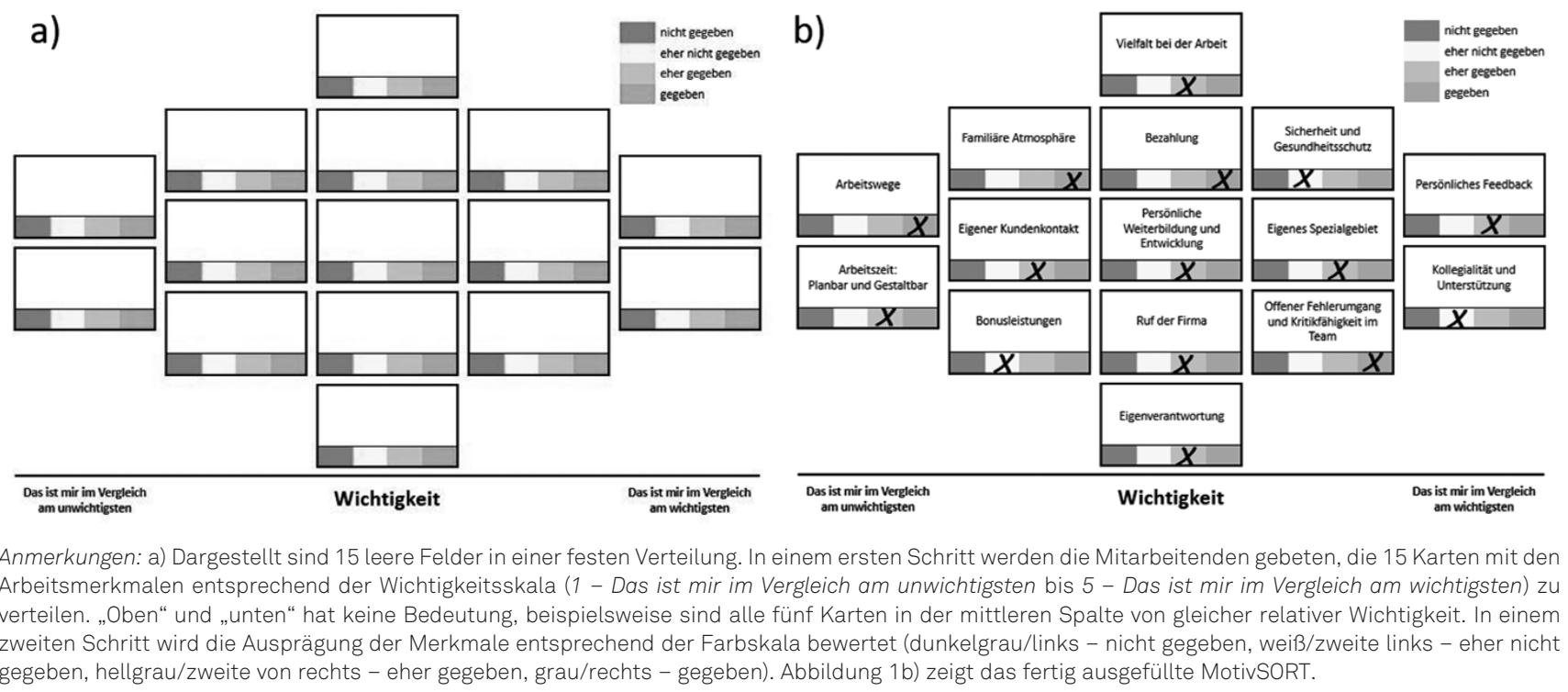

Abbildung 1. Leere und ausgefüllte Vorlage zur Bearbeitung von MotivSORT.

zen (Paulus, 2020). Hierzu müssen jedoch die individuellen Bedürfnisse der Mitarbeitenden bekannt und mit den Möglichkeiten des Unternehmens vereinbar sein. Mitarbeitendengespräche zwischen einzelnen Mitarbeitenden und der Führungskraft können hier den Rahmen zur notwendigen Reflexion und Diskussion bieten.

Als Unterstützung für diese bedürfnisorientierten Mitarbeitendengespräche wurde MotivSORT entwickelt. MotivSORT besteht aus einer Tischvorlage und 15 Karten, auf denen im Handwerk relevante Arbeitsmerkmale abgedruckt sind. Die Tischvorlage gibt eine feste Verteilung vor, auf der die Mitarbeitenden die Arbeitsmerkmale hinsichtlich der subjektiven Wichtigkeit bewerten sollen (Abbildung 1). In einem zweiten Schritt bewerten die Mitarbeitenden anhand der Farbskala die Ausprägung dieser Arbeitsmerkmale im aktuellen Unternehmen. Ziele der MotivSORT-gestützten Mitarbeitendengespräche sollten sein, (1) theoretisch fundierte, individuelle Ansatzpunkte zur Mitarbeitendenbindung zu erfassen (Screening), (2) Erwartungen, Möglichkeiten und Grenzen offen anzusprechen (Reflektionsgespräch) und (3) einen diagnostisch belastbaren Eindruck zu erhalten, wie der Betrieb in Bezug auf Bindung und Zufriedenheit zu bewerten ist (Organisationsdiagnostik). Das Instrument sollte einen einfachen Zugang zu berufsbezogenen Bedürfnissen und Arbeitsgestaltung für Führungskräfte und Mitarbeitende im Gespräch bieten, sprachlich leicht verständlich und in 15-20 Minuten durchführbar sein.

\section{Innovationsleistung}

\section{Instrument zur bedürfnisorientierten Arbeitsanalyse am Beispiel des Handwerks}

Drei Innovationsleistungen zeichnen das entwickelte Instrument MotivSORT aus. (1) Es werden theoretisch fundierte Erkenntnisse $\mathrm{zu}$ bindungsförderlichen Arbeitsmerkmalen anwendungsorientiert in die Praxis übertragen. (2) Auf diagnostischer Ebene soll MotivSORT Daten über die Bedürfnisse einzelner Mitarbeitender sowie deren Ausprägung im Betrieb liefern. Dabei wird eine vorgegebene Verteilung angewandt und die separate Erhebung der Dimensionen „Wichtigkeit der Arbeitsmerkmale“ und „Ausprägung der Merkmale im Betrieb“ umgesetzt. (3) Es werden empirisch fundierte Erkenntnisse zur mitarbeiterorientierten Führung praktisch anwendbar. Im persönlichen Gespräch können erfolgsrelevante Facetten des Führungsverhaltens, beispielsweise individuelle Unterstützung und bedürfnisorientierte Führung (Bass \& Avolio, 1990), im Betriebsalltag verankert werden.

Die skizzierten Innovationsleistungen basieren auf drei theoretischen und methodischen Konzepten der Arbeits- und Organisationspsychologie, die nachfolgend vorgestellt und auf MotivSORT übertragen werden sollen. 


\section{Konzeptueller Hintergrund}

\section{Bedürfnisorientierte Arbeitsgestaltung One Size Fits all?}

Im Instrument MotivSORT stellen theoretisch fundierte Arbeitsmerkmale die Grundlage zum Mitarbeitendengespräch und zur Diagnostik dar ${ }^{1}$. Um individuellen Bedürfnissen gerecht zu werden, werden diese - angelehnt an Konzepte der differentiellen Arbeitsgestaltung und Person-Umwelt-Passung - individuell in der Mitarbeitergesprächssituation gewichtet (Wichtigkeit) und bewertet (Ausprägung).

\section{Arbeitsmerkmale als Prädiktor von Bindung und Arbeitszufriedenheit}

Die Gestaltung von Arbeit wird in der Arbeits- und Organisationspsychologie als zentraler Weg zur Förderung von Bindung, Zufriedenheit und damit zur Fachkräftesicherung gesehen (Hacker \& Sachse, 2014; Hackman \& Oldham, 1975; Parker et al., 2017). Führungskräfte haben einen beachtlichen Einfluss auf die Arbeitsgestaltung, im Gegensatz $\mathrm{zu}$ schwer veränderbaren organisationalen Aspekten wie Kultur oder Unternehmensstruktur (Morgeson \& Humphrey, 2008). Der Gestaltungsspielraum erlaubt Führungskräften die Arbeit der Mitarbeitenden bedürfnisorientiert zu gestalten und so die Motivation, Zufriedenheit und Bindung der Mitarbeitenden zu erhöhen. Zugleich ist die Arbeitsgestaltung auch relevant für Performanz, Gesundheit, Lernen, Innovation, Prozessoptimierung und -anpassung (Parker, 2014). In Vorbereitung der Interviews mit Mitarbeitenden und Führungskräften, welche der schlussendlichen Instrumentenentwicklung dienten, wurden Theorien und Modelle der Arbeitspsychologie auf relevante Arbeitsmerkmale untersucht. Zentral sind hierbei vor allem die Arbeiten von Hackman \& Oldham (1975), welche als Grundlage der heutigen Arbeitsgestaltung angesehen werden kann, sowie die Ergänzungen um soziale Faktoren wie soziale Unterstützung von Morgeson \& Humphrey (2006). Beiden Modellen gemein sind die Arbeitsmerkmale Autonomie, Ganzheitlichkeit, Bedeutsamkeit der Aufgabe, Anforderungsvielfalt und Rückmeldung zur Tätigkeit, die aufgrund ihrer allgemeinen Gültigkeit Eingang in das zu entwickelnde Instrument finden sollten. Neben diesen konkreten Arbeitsmerkmalen geben weitere Modelle der Arbeitspsychologie Aufschluss über relevante Arbeitsmerkmale. Beispielhaft seien hier das Ge- rechtigkeitserleben (z.B. in Bezug auf Bezahlung, Folger \& Koronovsky, 1989), die Handlungsregulationstheorie (Hacker, 2005) oder die Theorie der sozialen Identität (z.B. in Bezug auf den Ruf der Firma; Tajfel \& Turner, 2004) zu nennen. Eine umfassende Auflistung der in MotivSORT enthaltenen Merkmale und ihrer theoretischen Entsprechungen findet sich in Tabelle E1.

Obwohl die genannten Arbeitsmerkmale für die Bindung und Zufriedenheit aller Mitarbeitenden wichtig sind, kann es zusätzliche Informationen liefern, auch auf persönliche Präferenzen der Mitarbeitenden zu achten (van Vianen, 2018). Hier spricht man von differentieller Arbeitsgestaltung, das heißt der Gestaltung von Arbeit unter Beachtung interindividueller Unterschiede.

\section{Differentielle Arbeitsgestaltung}

Die differentielle Arbeitsgestaltung erweitert die klassische Arbeitsgestaltung um die Dimension der individuellen Wichtigkeit (Ulich, 2010). Beispielsweise ist eine flexible Arbeitszeitgestaltung generell wünschenswert, für Mitarbeitende mit vielen familiären Verpflichtungen sind flexible Arbeitszeiten aber eventuell noch wichtiger. Die differentielle Arbeitsgestaltung wird auch in der PersonUmwelt-Passungs-Forschung (engl. Person-EnvironmentFit; Kristof-Brown \& Guay, 2011) untersucht. Die Umweltkomponente entspricht in diesem Projekt der Ausprägung der Arbeitsmerkmale (engl. supply), wie sie in der klassischen Arbeitsgestaltung untersucht wird. Hinzu kommt die Komponente der individuellen Wichtigkeit, also des individuell unterschiedlich starken Bedürfnisses (engl. need) nach dem jeweiligen Arbeitsmerkmal. In Abgleich von Ausprägung und Bedürfnis ergibt sich ein Passungsmaß (engl. need-supply-fit), welches positiv mit dem Erleben der Mitarbeitenden zusammenhängt. Demnach führt eine hohe Passung zwischen der Ausprägung eines Arbeitsmerkmals in der Umwelt (z. B. flexible Arbeitszeitgestaltung) und der individuellen Wichtigkeit für die Person (z. B. hohes Bedürfnis nach Flexibilität) zu einer stärkeren Bindung und Arbeitszufriedenheit. Diese und weitere Zusammenhänge der Passung mit Erlebensmaßen wie emotionaler Erschöpfung, Kündigung und Leistung konnten in zahlreichen Studien, Langzeituntersuchungen und Metaanalysen nachgewiesen werden (e.g., Tak, 2011; Taris \& Feij, 2001; van Vianen, 2018). Unter diesen Voraussetzungen erscheint eine Betrachtung der Arbeitsgestaltung auf den Dimensionen individuelle Wichtigkeit und wahrgenommene Ausprägung sinnvoll. 


\section{Umsetzung differentieller Arbeitsgestaltung in MotivSORT}

Wie gezeigt sind sowohl die Ausprägung eines Arbeitsmerkmals in der Umwelt als auch die individuelle Wichtigkeit dieses Merkmals für die Person von Bedeutung für die Bindung und Arbeitszufriedenheit von Mitarbeitenden. Dies stellt die erste Innovation des vorliegenden Projekts dar. Bekannte Messinstrumente der Arbeitsgestaltung erheben lediglich die Umweltkomponente (vgl. Job Diagnostics Survey (JDS), Hackman \& Oldham, 1975; Work Design Questionnaire (WDQ), Morgeson \& Humphrey, 2006). Dies ist kein Mangel oder Nachteil der genannten Erhebungsmethoden, sondern mit dem unterschiedlichen Anwendungskontext begründet. Bekannte Messinstrumente wie JDS und WDQ dienen hauptsächlich der Erhebung zu Forschungszwecken oder auf organisationaler Ebene. Das vorliegende Projekt hingegen hat zum Ziel, Mitarbeitendengespräche zu vereinfachen und individuelle Ansatzpunkte zur Arbeitsgestaltung zu identifizieren. Dies bedingt eine Erhebung der differentiellen Arbeitsgestaltung, d.h. eine Erhebung auf den Dimensionen Ausprägung und Wichtigkeit.

Aus den wissenschaftlichen Erkenntnissen der Arbeitsgestaltung ergibt sich eine Hürde für die Messung der Wichtigkeitsdimension: Die differentielle Arbeitsgestaltung geht zwar von individuellen Unterschieden in der Wichtigkeit aus, erkennt jedoch auch an, dass bis zu einem gewissen Grad alle Arbeitsmerkmale für alle Mitarbeitenden wichtig sind. Beispielsweise ist es höchst unwahrscheinlich, dass Mitarbeitende Merkmale wie Bezahlung, Eigenverantwortung oder Vielfalt als „unwichtig" bewerten, auch wenn sie nicht die wichtigsten Merkmale für sie sind. Daraus folgt für die Messung der Wichtigkeit mit üblicherweise genutzten absoluten Ratingskalen (z. B. Likertskalen; Likert, 1932) das Risiko von Deckeneffekten („Alle Merkmale sind mir wichtig.“). Für Unternehmen wäre dies insofern problematisch, dass eine Priorisierung von Merkmalen für die Arbeitsgestaltung („Welches ist das wichtigste Merkmal und sollte zuerst verbessert werden?") erschwert wird. Um diesem Problem vorzugreifen wird die Wichtigkeitsdimension in MotivSORT mit einer vorgegebenen Verteilung erfasst. Dies entspricht der zweiten Innovationsleistung im Projekt.

\section{Forced-Distribution-Ratingskalen zur Erfassung individueller Bedürfnisse}

Forced-Distribution-Ratingskalen (FDRS) sind relative Bewertungssysteme mit einer vorgegebenen Verteilung (Stewart et al., 2010). Das bedeutet, dass die zu bewer- tenden Merkmale nicht absolut bewertet werden (z. B. „XY ist mir nicht wichtig/wichtig."), sondern immer im Vergleich zu anderen Merkmalen (z.B. „XY ist mir im Vergleich zu den anderen Merkmalen nicht wichtig/wichtig.") Zudem müssen die Merkmale in einer vorgegebenen Verteilung angeordnet werden, d.h. nicht jedes Item kann mit der höchsten Wichtigkeit bewertet werden, wie es etwa bei Likertskalen der Fall ist.

Der Einsatz von FDRS bringt kontextübergreifend einige Vorteile mit sich. Aufgrund der vorgegebenen Verteilung werden Boden- und Deckeneffekte verhindert und somit die Varianz und die Interpretierbarkeit der Daten erhöht. Beispielsweise werden relative Ratingsysteme bei der Leistungsbewertung von Mitarbeitenden genutzt, um Deckeneffekte zu verhindern, d.h. alle oder die meisten Mitarbeitenden als "High-Performer" einzustufen (Stewart et al., 2010). So können z. B. wichtige Personalentscheidungen wie Beförderungen, Kündigungen oder Weiterbildungen leichter getroffen werden. Ein weiterer Vorteil von FDRS ist die intensive Auseinandersetzung mit den zu bewertenden Merkmalen (Albani et al., 2000). Aufgrund der Abhängigkeit der Bewertungen müssen die einzelnen Merkmale explizit gegeneinander abgewogen werden. Diese intensivere Auseinandersetzung begünstigt weiterführende Gespräche. Wenn Führungskräfte nach dem Einsatz von FDRS individuelle Auswertungsgespräche führen, kann dies den Mitarbeitenden eine offene und ehrliche Feedbackkultur signalisieren (Guralnik et al., 2004). Der Einsatz von FDRS ist somit nicht nur für die bedürfnisorientierte Arbeitsanalyse, sondern auch für darauffolgende Mitarbeitendengespräche sinnvoll.

\section{Umsetzung von FDRS in MotivSORT}

Durch den Einsatz von FDRS in MotivSORT können Deckeneffekte in der Merkmalsbewertung vermieden werden. Zudem zwingen FDRS die Mitarbeitenden zu einer stärkeren Auseinandersetzung mit dem Sachverhalt und begünstigen offene Gespräche und detaillierte Reflexionen der bedürfnisorientierten Arbeitsgestaltung im Austausch mit der Führungskraft. Hier setzt die dritte Innovationsleistung von MotivSORT an, da erfolgsrelevantes mitarbeiterorientiertes Führungsverhalten direkt umsetzbar wird.

\section{Mitarbeiterorientierte Führung}

Das Instrument MotivSORT soll neben der diagnostischen und gestalterischen Funktion auch mitarbeiterorientiertes Führungsverhalten umsetzbar und erlebbar machen. Gerade im unternehmerischen Alltag sind die ab- 
strakten Konzepte der psychologischen Führungsforschung nicht immer greifbar, obwohl die Führungsforschung auf die Bedeutung einer individuellen Beziehung zwischen Führungskraft und Geführten hinweist. So wird im Modell des umfassenden Führungsverhaltens (engl. Full Range of Leadership Model; Bass \& Avolio, 1990) individuelle Unterstützung (engl. individual consideration) als wichtige Komponente mitarbeiterorientierter Führung empirisch und metaanalytisch bestätigt. Auch das Vorgeben von Strukturen, also der klaren Rollen- und Aufgabenverteilung, ist ein zentraler Bestandteil mitarbeiterorientierter Führung (Schriesheim \& Bird, 1979). Im Kontrast zu aufgabenorientierter Führung, bei welcher die Effektivität und Effizienz betrieblicher Unternehmungen aktiv in den Vordergrund gestellt wird, gilt das Hauptaugenmerk mitarbeiterorientierter Führung dem Erleben (z. B. Tatkraft, Bindung) und Verhalten (z. B. Kündigung) der Mitarbeitenden (Felfe, 2009). Das Erzeugen dieser sogenannten „weichen“ Faktoren ist ein Prädiktor für „harte" Faktoren wie Produktivität und Fluktuation (Kovjanic et al., 2013). Negative Zusammenhänge zwischen mitarbeiterorientierter Führung und arbeitsbezogenem Stress, psychosomatischen Beschwerden und Burnout zeigen die gesundheitsbezogene Relevanz mitarbeiterorientierter Führung (Rowold \& Heinitz, 2008). Oftmals lassen sich Stressoren und Anforderungen der Arbeit nicht gänzlich verhindern, z.B. bei Schichtbetrieb oder Termindruck. Führung kann diesen Stressoren entgegenwirken und im Sinne eines Puffers die negativen Auswirkungen von teils unvermeidbaren hohen Anforderungen abmildern (Breevaart \& Bakker, 2018; Syrek et al., 2013). Aktuelle Studien sprechen dafür, dass die erfolgreiche Sicherung von Fachkräften eng damit verknüpft ist, wie gut Unternehmen auf die Erwartungen der Fachkräfte eingehen können (Schmidt et al., 2012). Unklar bleibt für Praktiker, wie genau mitarbeiterorientierte Führung im Unternehmen und im Zwiegespräch mit Mitarbeitenden umgesetzt werden kann. Hier setzt MotivSORT an und unterstützt Führungskräfte durch die Strukturierung von bedürfnisorientierten Mitarbeitendengesprächen.

\section{Beschreibung des Projektablaufs und der verwendeten Methoden}

Die Entwicklung gliederte sich in zwei Phasen. (1) In der Entwicklungsphase des Instruments MotivSORT mussten Arbeitsmerkmale definiert werden, die sowohl theoretisch fundiert als auch im Unternehmen praxisnah verständlich waren. (2) In der Pilotierungsphase wurden in zwei separaten Studien Aspekte der Validität und Akzeptanz in Betrieben der Handwerkskammern RheinlandPfalz evaluiert.

\section{Phase 1: Entwicklung und Aufbau von MotivSORT}

Die relevanten Arbeitsmerkmale wurden im Rahmen einer Interviewstudie erhoben, um so die Realitäten in den Betrieben möglichst genau abbilden zu können. Mittels eines standardisierten Interviews wurden Führungskräfte unterschiedlicher Hierarchien sowie Mitarbeitende zu relevanten Arbeitsmerkmalen befragt. Die Teilnehmenden wurden gefragt, warum Mitarbeitende gerne im aktuellen Betrieb arbeiten würden und mit welchen Eigenschaften ihres Betriebs sie eine aktuelle Stelle bewerben würden. Aus 16 Interviews konnten 114 Einzelnennungen identifiziert werden.

Entsprechend der induktiven Kategorienbildung (Mayring, 2015) wurden diese 114 Einzelnennungen in Expertendiskussionen (aus universitär-wissenschaftlicher Expertise seitens der psychologischen Diagnostik und langjährigen Betriebsberatern der Handwerkskammern) durch Zusammenführung doppelter Nennungen, Exklusion nicht relevanter Merkmale (z. B. Identifikation mit dem Job, welches ein Ergebnis und kein Merkmal guter Arbeitsgestaltung ist; vgl. Trépanier et al., 2015), sowie Zusammenführung in übergeordnete Kategorien zu 15 Arbeitsmerkmalen zusammengefasst. Die resultierenden 15 Arbeitsmerkmale sind: Arbeitswege, Arbeitszeit: Planbar und Gestaltbar, Bezahlung, Bonusleistungen, Eigener Kundenkontakt, Eigenes Spezialgebiet, Eigenverantwortung, Familiäre Atmosphäre, Kollegialität und Unterstützung, Offener Fehlerumgang und Kritikfähigkeit im Team, Persönliches Feedback, Persönliche Weiterbildung, Ruf der Firma, Sicherheit und Gesundheitsschutz sowie Vielfalt bei der Arbeit. Diese 15 Arbeitsmerkmale werden mit MotivSORT bewertet. Dazu wird jedes Merkmal den Mitarbeitenden auf einer Legekarte präsentiert, die im späteren Verlauf auf einer Tischvorlage zur Bewertung abgelegt werden kann. Zur genaueren Definition der Merkmale und für eine erhöhte Verständlichkeit für die Mitarbeitenden wurden auf die Rückseite der Karten beispielhafte Inhalte gedruckt, die Einzelnennungen der Erhebung entsprechen. Beispielsweise sind die Einzelnennungen Kollegen und Firma helfen bei Fragen und Problemen, Unterstützung und gegenseitiges Helfen (z.B. bei Krankheit usw.) und gegenseitiges Aushelfen und Tipps von Kollegen auf der Rückseite der Legekarte Kollegialität und Unterstützung abgedruckt. Neben den 15 Legekarten erhalten die Mitarbeitenden eine Tischvorlage (ca. 30 × $60 \mathrm{~cm}$ ), auf der die Bewertung der Arbeitsmerkmale erfolgt (vgl. Abbildung 1). Die Vorlage gibt, entsprechend dem FDRS-Ansatz, eine fixierte Verteilung der Merkmale bezüglich der individuellen Wichtigkeit vor. Die Mitarbeitenden sind zunächst gefragt, die 15 Arbeitsmerkmale hinsichtlich der relativen Wichtigkeit 
zu bewerten. Die Skala reicht von „1- Das ist mir im Vergleich am unwichtigsten“ bis , 5 - Das ist mir im Vergleich am wichtigsten". Dabei müssen die Mitarbeitenden einer vorgegebenen Verteilung folgen (vgl. Abbildung 1). Es können beispielsweise nur zwei Merkmale mit 1 oder 5 bewertet werden (linke bzw. rechte Spalte). Nach der Bewertung der Wichtigkeit erfolgt die Bewertung der subjektiv empfundenen Ausprägung dieses Merkmals im Unternehmen. Dies erfolgt anhand einer vierstufigen Farbskala unter den Kartenfeldern (,nicht gegeben“ (rot), "eher nicht gegeben“ (gelb), ,eher gegeben“ (hellgrün), "gegeben“ (dunkelgrün). Die gesamte Durchführung von MotivSORT ist in 10 Minuten möglich. In einem Nachgespräch unter vier Augen können individuelle Veränderungen, Möglichkeiten der persönlichen Weiterentwicklung und Grenzen der betrieblichen Machbarkeit besprochen werden. Zur Gesprächsführung steht der Führungskraft ein Reflexionsleitfaden zur Verfügung. Diese Erkenntnisse können zudem auf Team- oder Betriebsebene betrachtet werden (Betriebsmonitor ${ }^{2}$ ), um den unternehmensweiten IST-Zustand der Bewertung von Arbeitsmerkmalen zu erfassen und Maßnahmen der Organisationsentwicklung abzuleiten.

\section{Phase 2: Akzeptanz und Validität von MotivSORT}

Es wurden zur Evaluation der Akzeptanz und Validität zwei Studien durchgeführt. Studie 1 fand im Bereich der Handwerkskammer Trier statt (Fokus Kriteriumsvalidität und Akzeptanz) und Studie 2 im Bereich der Handwerkskammern Rheinland-Pfalz (Fokus Kriteriums- und Konvergenzvalidität).

\section{Methode}

\section{Ablauf der Studien}

Studie 1. Nach Entwicklung von MotivSORT in Phase 1 wurde dessen Akzeptanz aus Sicht der Mitarbeitenden und die Kriteriumsvalidität in Bezug auf organisationsrelevante Außenkriterien mit Bezug zur Fachkräftesicherung (z. B. Bindung, Arbeitszufriedenheit, Kündigungsabsicht) untersucht. Alle Erhebungen fanden in den jeweiligen Betrieben während der Arbeitszeit in separaten und ruhigen Räumlichkeiten statt. Alle Erhebungen wurden durch einen geschulten Erhebungsleiter durchgeführt. Die teilnehmenden Mitarbeitenden bearbeiteten zunächst MotivSORT. Danach füllten sie einen Fragebogen zum berufsbezogenen Erleben und zur Akzeptanz des Verfahrens aus. Insgesamt dauerte die Erhebung $30 \mathrm{Mi}-$ nuten.

Studie 2. Im Rahmen einer landesweiten Studie im Handwerk Rheinland-Pfalz wurde die Validität in einer zweiten, unabhängigen Stichprobe erneut geprüft. Die Erhebungen wurden von mehreren Mitarbeitenden der Handwerkskammern durchgeführt. Die durchführenden Mitarbeitenden der Handwerkskammern wurden in einer eintägigen Schulung in Grundlagen der Diagnostik und der Anwendung von MotivSORT geschult. Die Mitarbeitenden bearbeiteten in der Erhebungssituation MotivSORT sowie einen zugehörigen Fragebogen zum berufsbezogenen Erleben (ca. 30 min).

\section{Stichprobe}

Studie 1. Es nahmen $N=51$ Mitarbeitende aus acht Betrieben in sieben Gewerken im Zugehörigkeitsbereich der Handwerkskammer Trier teil. Die Betriebe hatten eine Größe von drei bis 12 Mitarbeitenden. Die Mitarbeitenden waren zwischen 18 und 60 Jahren $(M=36.08, S D=13.83)$ alt und zwischen einem Monat und 31.00 Jahren im Unternehmen tätig $(M=6.98, S D=6.76) .54 .90 \%$ der Beschäftigten waren weiblich.

Studie 2. Es nahmen $N=203$ Mitarbeitende aus $12 \mathrm{Be}$ trieben in neun Gewerken im Zugehörigkeitsbereich der Handwerkskammern Rheinland-Pfalz teil. Die Betriebe hatten eine Größe von fünf bis 42 Mitarbeitenden. Die Mitarbeitenden waren zwischen 16 und 69 Jahren $(M=$ 37.84; $S D=13.77)$ alt und zwischen einem Monat und 45.25 Jahren im Unternehmen tätig $(M=9.01$ Jahre; $S D=$ 9.17). $32.20 \%$ der Beschäftigten waren weiblich.

\section{Verwendete Skalen zur Erfassung von Akzeptanz und Validität}

Mithilfe eines Fragebogens wurden Mitarbeitende nach der Nutzung von MotivSORT bezüglich (1) der Nützlichkeit von MotivSORT, (2) ihrem arbeitsbezogenen Erleben und (3) ihrer arbeitsbezogenen Motiverfüllung befragt. Mithilfe dieser Skalen wird (1) die Akzeptanz von MotivSORT, (2) die Kriteriumsvalidität und (3) die Konvergenzvalidität untersucht. Die Bewertung der Aussagen 
erfolgte, wenn nicht anders vermerkt, auf einer bipolaren, sechsstufigen Bewertungsskala (-3 = trifft überhaupt nicht $z u,-2=$ trifft nicht $z u,-1=$ trifft eher nicht $z u, 1=$ trifft eher $z u, 2=$ trifft $z u, 3=$ trifft voll $z u$ ).

Die Akzeptanz von MotivSORT seitens der Mitarbeitenden wurde mit sechs Items (z.B. wahrgenommene Nützlichkeit, Jobrelevanz) auf Grundlage des Technologie-Akzeptanz-Modells (TAM2; Venkatesh \& Davis, 2000) erhoben. Zur Überprüfung der Kriteriumsvalidität wurde das arbeitsbezogene Erleben der Mitarbeitenden erhoben. Es wurden affektive (affektive Bindung an die Organisation, Arbeitszufriedenheit), motivationale (emotionale Erschöpfung, Tatkraft) und kognitive Maße (Abschalten von der Arbeit, Identifikation, subjektive körperliche Gesundheit) sowie Kündigungsabsicht als Verhaltensmaß erhoben. Die Überprüfung der Konstruktvalidität erfolgte durch den Abgleich von MotivSORT mit einer etablierten Skala zur Erfassung berufsbezogener Bedürfnisse (WBNS; van den Broeck et al., 2010) ${ }^{3}$.

\section{Datenauswertung und -analyse}

MotivSORT erhebt 15 Arbeitsmerkmale als formative Indikatoren (vgl. Ellwart \& Konradt, 2011). Daher erfolgt die Prüfung der Validität von MotivSORT mithilfe formativreflektiver Partial-Least-Squares-Strukturgleichungsmodelle, bzw. im Fall von Arbeitszufriedenheit mithilfe eines formativ-formativen Partial-Least-Squares-Strukturgleichungsmodells. Alle Analysen wurden mit SmartPLS 3 (Ringle et al., 2015) durchgeführt. Vorab wurden die Dimensionen Wichtigkeit und Ausprägung pro Arbeitsmerkmal als Produkt verrechnet, sodass pro Arbeitsmerkmal ein Indikator in die Modelle einfloss. Zur genaueren Betrachtung der Entscheidungsregeln zur Wahl formativer und reflektiver Messmodelle sei auf die Werke von Hair und Kollegen (2017) sowie Weiber und Mühlhaus (2014) verwiesen.

\section{Ergebnisse Studie 1}

\section{Akzeptanz von MotivSORT}

Insgesamt zeigt sich eine sehr positive Bewertung sämtlicher Dimensionen der Akzeptanz ${ }^{4}$. Die Mittelwerte bewegen sich auf der sechsstufigen Skala $(-3=$ trifft nicht $z u$ bis 3 = trifft voll $z u$ ) zwischen $M=1.65$ (Nutzungsintenti- on) und $M=2.40$ (Wahrgenommene Einfachheit der Nutzung). Die wahrgenommene Nützlichkeit und die wahrgenommene Einfachheit der Nutzung wurden in 98.0 \% der Fälle als positiv (min. 1 = trifft eher $z u$ ) bewertet. $96.1 \%$ der Mitarbeitenden gaben an, dass MotivSORT bei Führungskräften und Kollegen gut ankommen würde (subjektive Norm). 90.2\% der Mitarbeitenden bewerteten den Einsatz des MotivSORT als jobrelevant. Insgesamt würden $92.2 \%$ der Mitarbeitenden MotivSORT gerne in ihrem Unternehmen nutzen. Die Akzeptanz von MotivSORT seitens der Mitarbeitenden ist damit auf sämtlichen Dimensionen als sehr hoch zu bewerten.

\section{Kriteriumsvalidität}

Es zeigten sich signifikant positive Zusammenhänge für die erfassten Kriterien (siehe Tabelle 1). Mitarbeitende mit einer hohen Ausprägung der erfragten Arbeitsmerkmale (hoher MotivSORT-Wert) waren enger an das Unternehmen gebunden $(\beta=.78, p<.001)$, hatten eine höhere Arbeitszufriedenheit $(\beta=.87, p=.032)$ und Tatkraft $(\beta=.80, p<.001)$, waren emotional weniger erschöpft $(\beta=-.58, p<.001)$ und hatten eine geringere Kündigungsintention $(\beta=-.69, p<.001)$.

\section{Ergebnisse Studie 2}

\section{Kriteriumsvalidität}

Mitarbeitende mit einer hohen Ausprägung der erfragten Arbeitsmerkmale (hoher Motiv SORT-Wert) zeigten erneut eine stärkere Bindung an das Unternehmen $(\beta=.71$, $p<.001)$, eine höhere Arbeitszufriedenheit $(\beta=.56, p<$ $.001)$ und Tatkraft $(\beta=.52, p<.001)$ sowie eine geringere Kündigungsintention $(\beta=-.52, p<.001)$. Die Ergebnisse aus Studie 1 konnten somit repliziert werden. Zusätzlich gaben die Mitarbeitenden an, dass sie bei stärkerer Ausprägung der mit MotivSORT erfragten Arbeitsmerkmale auch eine stärkere Identifikation mit dem Unternehmen haben $(\beta=.61, p<.001)$. Die Zusammenhänge mit subjektiver körperlicher Gesundheit und dem Abschalten von der Arbeit wurden nicht signifikant.

\footnotetext{
Die detaillierte Darstellung der verwendeten Skalen findet sich im ESM 3.

4 Detailliertere Ergebnisse der Akzeptanzbewertungen können im ESM 4 eingesehen werden.
} 
Tabelle 1. Validierung von MotivSORT. Regressionskoeffizienten von MotivSORT mit berufsbezogenen Maßen des Erlebens sowie Vergleich dieser Zusammenhänge mit den Zusammenhängen eines reflektiven Maßes berufsbezogener Bedürfniserfüllung (W-BNS)

\begin{tabular}{|c|c|c|c|c|c|c|}
\hline \multirow[b]{3}{*}{ Erlebensmaße } & \multirow{2}{*}{\multicolumn{2}{|c|}{$\begin{array}{c}\text { Studie } 1 \\
\text { MotivSORT }\end{array}$}} & \multicolumn{4}{|c|}{ Studie 2} \\
\hline & & & \multicolumn{2}{|c|}{ MotivSORT } & \multicolumn{2}{|c|}{ W-BNS } \\
\hline & $\beta$ & $R^{2}$ & $\beta$ & $R^{2}$ & $R^{2}$ & $\Delta^{1}$ \\
\hline Bindung & $.78 * \star \star$ & $60.5 \%$ & $.71 * \star \star$ & $50.5 \%$ & $34.8 \%$ & 15.7 \\
\hline Arbeitszufriedenheit & $.87^{\star}$ & $74.9 \%$ & $.56^{\star \star \star}$ & $31.7 \%$ & $35.9 \%$ & -4.2 \\
\hline Tatkraft & $.80 * \star \star$ & $63.5 \%$ & $.52 * \star *$ & $27.1 \%$ & $29.6 \%$ & -2.5 \\
\hline Emotionale Erschöpfung & $-.58 * \star \star$ & $33.1 \%$ & - & - & - & - \\
\hline Kündigungsabsicht & $-.69 * \star \star$ & $47.9 \%$ & $-.52 \star * \star$ & $27.0 \%$ & $20.7 \%$ & 6.3 \\
\hline Körperliche Gesundheit & - & - & .29 & - & - & - \\
\hline Abschalten von der Arbeit & - & - & .33 & - & - & - \\
\hline Identifikation & - & - & $.61 * \star *$ & $37.0 \%$ & $24.1 \%$ & 12.9 \\
\hline
\end{tabular}

Anmerkungen: $* * \star p<.001, * p<.05 N_{\text {Studie1 }}=51, N_{\text {Studiez }}=203 .{ }^{1} \Delta$ (Delta) beschreibt den Unterschied der aufgeklärten Varianz $\left(R^{2}\right)$ zwischen MotivSORT und W-BNS in Prozentpunkten. Positive Werte entsprechen einer höheren Varianzaufklärung durch MotivSORT. Alle berichteten Zusammenhänge des Erlebens mit der W-BNS waren auf dem *** $p<.001-$ Niveau signifikant

\section{Konvergenzvalidität}

Das Erleben der Mitarbeitenden hängt vergleichbar stark mit den erhobenen Arbeitsmerkmalen (MotivSORT) und der Erfüllung beruflicher Grundbedürfnisse (W-BNS) zusammen (Tabelle 1). Somit kann MotivSORT bezüglich der Aussagekraft über das Mitarbeitendenerleben mit etablierten Skalen mithalten. Bezüglich der Bindung, der Identifikation und der Kündigungsintention zeigten sich deutliche Vorzüge von MotivSORT. MotivSORT erklärte hier 6.3 bis 15.7 Prozentpunkte mehr Varianz als die WBNS. Bezüglich Arbeitszufriedenheit und Tatkraft klärte die W-BNS mehr Varianz auf.

\section{Diskussion}

Die hohe Akzeptanz seitens der Mitarbeitenden, die Vergleichbarkeit mit ähnlichen, etablierten Skalen (Konvergenzvalidität) sowie die Zusammenhänge mit Erlebensmaßen (Kriteriumsvalidität) weisen MotivSORT als valide und nützlich aus. Die untersuchten Arbeitsmerkmale weisen teilweise hohe Zusammenhänge mit arbeitsbezogenem affektiven, motivationalen und kognitiven Erleben der Mitarbeitenden auf. Diese Zusammenhänge sind vergleichbar mit den Zusammenhängen des Erlebens mit der Erfüllung von arbeitsbezogenen Grundbedürfnissen, d.h. die Arbeitsanalyse mit MotivSORT klärt ähnlich viel Varianz im Erleben der Mitarbeitenden auf wie die Analyse der Bedürfniserfüllung (Konvergenzvalidität). Die Arbeitsanalyse mit MotivSORT ist ökonomisch, da sie in kurzer Zeit indirekte Rückschlüsse auf die Bindung und Zufriedenheit der Mitarbeitenden zulässt. Mit Motiv-
SORT können zudem direkte Ableitungen für die Arbeitsgestaltung auf individueller und organisationaler Ebene getroffen werden. Die hohe Akzeptanz seitens der Mitarbeitenden zeigt, dass MotivSORT nicht nur valide ist, sondern auch in der Anwendung im Unternehmen gern genutzt wird.

Trotz hoher Praxisrelevanz des Instruments und der Ergebnisse soll an dieser Stelle auf wissenschaftliche Grenzen hingewiesen und zukünftige Forschungsthemen adressiert werden. (1) MotivSORT erfasst die Ausprägung von Arbeitsmerkmalen auf formative Weise. Formative Messmodelle sind immer nur so gültig wie ihre Indikatoren. Die Vollständigkeit der Indikatoren muss demnach bei der Übertragung von MotivSORT in andere Branchen überprüft werden. (2) In MotivSORT werden Arbeitsmerkmale aufgrund ökonomischer Überlegungen per Single Item erfasst. Nachteile der Messung mit Single Items (z. B. kognitiv hoher Anspruch, Abstraktheit, enge Konstruktdefinition; vgl. Weiber \& Mühlhaus, 2014) werden durch die Ergänzung von Verhaltensindikatoren abgeschwächt. Eventuell unterschiedliche Auslegungen der Arbeitsmerkmale sind erwünscht und können im nachfolgenden Gespräch offenbar werden. Dennoch können zukünftige Untersuchungen zur Definitionsbreite der Arbeitsmerkmale, bspw. mit der Methode des lauten Denkens (Ericsson \& Simon, 1980), hilfreich bei der weiteren Entwicklung sein und zur Identifikation eventuell fehlender (Teil)Indikatoren beitragen. (3) In den durchgeführten Studien wurden die Werte für Wichtigkeit und Ausprägung der Arbeitsmerkmale als Produkt verrechnet. Dahinter steckt die Annahme, dass höhere Werte generell positive Auswirkungen auf das Erleben der Mitarbeitenden haben (vgl. Parker et al., 2017). Die Verrechnung der Dimensionen bringt Nachteile mit sich, da nicht mehr 
nachvollzogen werden kann, ob die Varianzaufklärung der Erlebensmaße auf die Bewertung der Wichtigkeit oder die Bewertung der Ausprägung zurückzuführen ist. Diese Information wäre jedoch für Unternehmen hilfreich, um zu wissen, ob sie eher auf allgemeine oder differentielle Arbeitsgestaltung setzen sollten. Zukünftige Forschung könnte diese unabhängigen Effekte mithilfe polynomialer Regressionsanalysen (Dörendahl et al., 2020) untersuchen und so die Aussagekraft von MotivSORT weiter erhöhen. (4) Aufgrund der querschnittlichen Durchführung der Studien können lediglich Zusammenhänge mit dem Erleben der Mitarbeitenden berichtet werden. Mit Blick auf den Forschungsstand ist anzunehmen, dass die Passung von Person und Organisation einen kausalen Effekt auf das Erleben der Mitarbeitenden hat (Trépanier et al., 2015; van den Broeck et al., 2010). Von ähnlichen Ergebnissen in Längsschnittstudien unter der Anwendung von MotivSORT ist, auch aufgrund der gezeigten Konvergenzvalidität, auszugehen. Neben den genannten Forschungsperspektiven gibt es zahlreiche Anwendungsperspektiven und Übertragungsmöglichkeiten für den Einsatz von MotivSORT, auf die im nächsten Abschnitt eingegangen werden soll.

\section{Anwendungsperspektiven und Übertragungsmöglichkeiten in andere Bereiche}

MotivSORT wurde entwickelt, um Führungskräfte im Handwerk bei der mitarbeiterorientierten Führung und bedürfnisorientierten Arbeitsgestaltung zu unterstützen. Hierbei wird MotivSORT genutzt, um eine Arbeitsanalyse auf individueller und unternehmensweiter Ebene durchzuführen und daraus Ableitungen für die Personal- und Organisationsentwicklung $\mathrm{zu}$ gewinnen. Neben dem Zweck der Personal- und Organisationsentwicklung ist MotivSORT auch auf andere Branchen und Themenbereiche übertragbar und wird seit 2021 durch Moldzio \& Partner - Institut für Personalauswahl insbesondere für die Nutzung im eignungsdiagnostischen Kontext weiterentwickelt.

\section{Branchengeneralität}

MotivSORT wurde als diagnostisches Instrument im Handwerk entwickelt und zunächst branchenspezifisch eingesetzt. Die zugrundeliegende Methodik (FDRS; Stewart et al., 2010) sowie die generelle Relevanz von
Merkmalen der Arbeitsgestaltung spricht jedoch für eine hohe Anwendbarkeit in anderen Branchen. Vor allem Berufe und Branchen, in denen ähnlich zum Handwerk eine hohe Interaktionsfrequenz mit Teammitgliedern, Führungskräften und Externen besteht, könnten MotivSORT ohne komplette Neukonstruktion in seiner aktuellen Form vortesten und bei Bedarf anpassen.

\section{MotivSORT in der Personalarbeit}

Neben dem standardmäßigen Einsatz von MotivSORT in der Personalentwicklung, beispielsweise im Rahmen von Entwicklungsgesprächen, können Führungskräfte MotivSORT auch anderweitig in der Personalarbeit einsetzen. Aus dem Abgleich individueller Bedürfnisse und betrieblicher Gegebenheiten ergeben sich auch innerbetriebliche Stärken, beispielsweise wenn alle oder die meisten Mitarbeitenden einzelne Facetten des Unternehmens als durchweg gegeben ansehen. In der Validierungsstichprobe aus dem Handwerk ist dies beispielsweise für das Merkmal „Kollegialität und Unterstützung“ der Fall. Dieses Wissen können Führungskräfte nutzen, um Ihre Stellenanzeigen spezifischer zu formulieren und so genau die Interessierten anzusprechen, die in das Firmenprofil passen. Ebenso können Bewerberinnen und Bewerber, die die fachlichen Anforderungen erfüllen, nach Ihren Bedürfnissen befragt und diese mit den betrieblichen Gegebenheiten abgeglichen werden. MotivSORT könnte hier im Sinne einer realistischen Tätigkeitsvorschau eingesetzt werden, um auch persönlich passende Bewerberinnen und Bewerber zu identifizieren, die dann mit höherer Wahrscheinlichkeit länger im Unternehmen verbleiben und so kostspielige frühzeitige Abgänge verhindern (Phillips, 1998).

\section{Einsatz in anderen Themenbereichen}

Die für MotivSORT angewandte Methodik der FDRS in Kombination mit einer Abfrage von Bedürfnissen und Gegebenheiten (Person-Umwelt-Passung) ist theoretisch auf andere Themenbereiche übertragbar. So könnten Themen wie betriebliches Gesundheitsmanagement (z. B. durch Screening-/Orientierungsverfahren), Teamentwicklung oder Veränderungsprozesse vom Einsatz partizipativer Instrumente profitieren. Beispielsweise erhöht die Einbindung von Mitarbeitenden in Veränderungsprozesse deren Vertrauen und Akzeptanz für später folgende Maßnahmen (Morgan \& Zeffane, 2003). So könnte der Ansatz von MotivSORT zur Bewertung von Widerständen 
in Veränderungsprozessen eingesetzt werden oder der Betriebsmonitor als visueller Reflexionsgegenstand im Veränderungsprozess dienen (vgl. Ellwart et al., 2016). Aufgrund der häufig sehr individuellen Prozesse empfiehlt sich hier, ähnlich wie in der initialen Entwicklung von MotivSORT im Handwerk, eine projektbezogene Entwicklung, die einen konkreten Nutzen beziehungsweise Anwendungskontext des benötigten Instruments vorgibt.

\section{Elektronische Supplemente (ESM)}

Die elektronischen Supplemente sind mit der OnlineVersion dieses Artikels verfügbar unter https://doi.org/ 10.1026/0932-4089/a000380

ESM 1. Tabelle E1. Arbeitsmerkmale in MotivSORT und deren Entsprechungen im JCM, WDQ und weiteren Theorien und Modellen der (Arbeits-)Psychologie

ESM 2. Darstellung eines Betriebsmonitors

ESM 3. Verwendete Skalen zur Erfassung von Akzeptanz und Validität

ESM 4. Tabelle E2. Bewertung der Akzeptanz von MotivSORT seitens der Mitarbeitenden

\section{Literatur}

Albani, C., Blaser, G., Jacobs, U., Jones, E., Geyer, M. \& Kächele, H. (2000). Die Methode des „Psychotherapie-Prozess Q-Sort“. Zeitschrift für Klinische Psychologie, Psychotherapie und Psychiatrie, 48, $151-171$.

Bass, B. M. \& Avolio, B. (1990). Transformational leadership development. Manual for the multifactor leadership questionnaire. Palo Alto, CA: Consulting Psychologists.

Breevaart, K. \& Bakker, A. B. (2018). Daily job demands and employee work engagement: The role of daily transformational leadership behavior. Journal of Occupational Health Psychology, 23, 338 - 349. https://doi.org/10.1037/ocp0000082

Dörendahl, J, Niepel, C. \& Greiff, S. (2020). Actually getting some satisfaction on the job: Need-supply fit of fundamental motives at work. Frontiers in Psychology, 11. https://doi.org/10.3389/ fpsyg.2020.01740

Ellwart, T. \& Konradt, U. (2011). Formative versus reflective measurement: an illustration using work-family balance. The Journal of Psychology, 145, 391 -417. https://doi.org/10.1080/ 00223980.2011 .580388

Ellwart, T., Peiffer, H., Matheis, G. \& Happ, C. (2016). Möglichkeiten und Grenzen eines Online Team Awareness Tools (OnTEAM) in Adaptationsprozessen. Zeitschrift für Wirtschaftspsychologie, 4, 5-15. https://doi.org/10.1007/978-3-662-55689-4

Ericsson, K. A. \& Simon, H. A. (1980). Verbal Reports as Data. Psychological Review, 87, 215-251.

Felfe, J. (2009). Mitarbeiterführung (Praxis der Personalpsychologie $\mathrm{Bd}$. 20). Göttingen: Hogrefe.
Folger, R. \& Konovsky, M. (1989). Effects of procedural and distributive justice on reactions to pay raise decisions. Academy of Management Journal, 32, 115-130.

Guralnik, O., Rozmarin, E. \& So, A. (2004). Forced Distribution: Is It Right for You? Human Resource Development Quarterly, 15, $339-345$.

Hacker, W. (2005). Allgemeine Arbeitspsychologie. Psychische Regulation von Wissens-, Denk- und körperlicher Arbeit (2. Aufl.). Bern: Huber.

Hacker, W. \& Sachse, P. (2014). Allgemeine Arbeitspsychologie: Psychische Regulation von Tätigkeiten (3., vollst. überarb. Aufl.). Göttingen: Hogrefe.

Hackman, J. R. \& Oldham, G. R. (1975). Development of the job diagnostic survey. Journal of Applied Psychology, 60, 159-170.

Hair, J. F., Hult, G. T. M., Ringle, C. M., Sarstedt, M., Richter, N. F. \& Hauff, S. (2017). Partial Least Squares Strukturgleichungsmodellierung: Eine anwendungsorientierte Einführung. München: Franz Vahlen.

Kovjanic, S., Schuh, S. C. \& Jonas, K. (2013). Transformational leadership and performance: An experimental investigation of the mediating effects of basic needs satisfaction and work engagement. Journal of Occupational and Organizational Psychology, 86, 543-555. https://doi.org/10.1111/joop.12022

Kristof-Brown, A. \& Guay, R. P. (2011). Person-environment fit. In S. Zedeck (Ed.), APA handbook of industrial and organizational psychology, Vol 3: Maintaining, expanding, and contracting the organization (pp. 3-50). Washington, DC, US: American Psychological Association. https://doi.org/10.1037/12171-001

Likert, R. (1932). A technique for the measurement of attitudes. Archives of Psychology, 22 140, 55.

Mayring, P. (2015). Qualitative Inhaltsanalyse: Grundlagen und Techniken (12. Aufl.). Weinheim. Beltz.

Morgan, D. \& Zeffane, R. (2003). Employee involvement, organizational change and trust in management. The International Journal of Human Resource Management, 14(1), 55-75. https://doi.org/10.1080/09585190210158510

Morgeson, F. P. \& Humphrey, S. E. (2006). The Work Design Questionnaire (WDQ): developing and validating a comprehensive measure for assessing job design and the nature of work. The Journal of applied psychology, 91, 1321-1339. https://doi.org/ 10.1037/0021-9010.91.6.1321

Morgeson, F. P. \& Humphrey, S. E. (2008). Job and team design: Toward a more integrative conceptualization of work design. Research in Personnel and Human Resource Management, 27, 39 -91. https://doi.org/10.1016/S0742-7301(08)27002-7

Parker, S. K. (2014). Beyond motivation: job and work design for development, health, ambidexterity, and more. Annual review of psychology, 65, 661-691. https://doi.org/10.1146/annurevpsych-010213-115208

Parker, S. K., Morgeson, F. P. \& Johns, G. (2017). One hundred years of work design research: Looking back and looking forward. The Journal of applied psychology, 102(3), 403-420. https://doi.org/10.1037/apl0000106

Paulus, L. (2020). Attraktives Handwerk - motivierte Mitarbeiter. In M. Schmitz \& R. Schmidpeter (Hrsg.), CSR in Rheinland-Pfalz (S. 497 - 499). Heidelberg: Springer.

Phillips, J. M. (1998). Effects of Realistic Job Previews on Multiple Organizational Outcomes: A Meta-Analysis. Academy of Management Journal, 41, 673-690. https://doi.org/10.5465/256964

Ringle, C. M., Wende, S. \& Becker, J.-M. (2015). SmartPLS 3 [Computer Software]. SmartPLS GmbH. Verfügbar unter: www. smartpls.com

Rowold, J. \& Heinitz, K. (2008). Führung als Stressbarrieren: Zum Zusammenhang zwischen transformationaler, transaktionaler, mitarbeiter- und aufgabenorientierter Führung und Indikatoren 
von Stress bei Mitarbeitern. Zeitschrift für Personalpsychologie, 7, $129-140$.

Schirner, S., Malin, L., Hickmann, H. \& Werner, D. (2021). Fachkräfteengpässe in Unternehmen: Fachkräftemangel und Nachwuchsqualifizierung im Handwerk. Köln: Institut der deutschen Wirtschaft. Verfügbar unter: http://hdl.handle.net/10419/233865

Schmidt, K., Meyer, J. E., Liebeneiner, J., Schmidt, C. E. \& Hüttenbrink, K. B. (2012). Fachkräftemangel in Deutschland: Umfrage zu Erwartungen von Chefärzten an junge Mitarbeiter. HNO, 60(2), 102 - 108. https://doi.org/10.1007/s00106-011-2406-9

Schriesheim, C. A. \& Bird, B. J. (1979). Contributions of the Ohio State Studies to the Field of Leadership. Journal of Management, 5(2), $135-145$.

Stewart, S. M., Gruys, M. L. \& Storm, M. (2010). Forced distribution performance evaluation systems: Advantages, disadvantages and keys to implementation. Journal of Management \& Organization, 16(1), 168-179. https://doi.org/10.1017/S18333672 00002340

Syrek, C. J., Apostel, E. \& Antoni, C. H. (2013). Stress in highly demanding IT jobs: transformational leadership moderates the impact of time pressure on exhaustion and work-life balance. Journal of occupational health psychology, 18(3), 252-261. https://doi.org/10.1037/a0033085

Tajfel, H. \& Turner, J. C. (2004). The Social Identity Theory of Intergroup Behavior. In J. T. Jost \& J. Sidanius (Eds.), Political psychology: Key readings (pp. 293). New York, NY: Psychology Press. https://doi.org/10.4324/9780203505984-16

Tak, J. (2011). Relationships between various person-environment fit types and employee withdrawal behavior: A longitudinal study. Journal of Vocational Behavior, 78, 315-320. https://doi. org/10.1016/j.jvb.2010.11.006

Taris, R. \& Feij, J. A. (2001). Longitudinal Examination of the Relationship Between Supplies-Values Fit and Work Outcomes. Applied Psychology: An International Review, 50(1), 52-80.

Thomä, J. (2014). Fachkräftemangel im Handwerk? - Eine Spurensuche. WSI-Mitteilungen, 67(8), 590 -598. https://doi.org/ 10.5771/0342-300X-2014-8-590

Trépanier, S.-G., Fernet, C. \& Austin, S. (2015). A longitudinal investigation of workplace bullying, basic need satisfaction, and employee functioning. Journal of occupational health psychology, 20(1), 105 -116. https://doi.org.10.1037/a0037726

Ulich, E. (2010). Aufgabengestaltung. In U. Kleinbeck, K.-H. Schmidt \& N.-P. Birbaumer (Hrsg.), Arbeitspsychologie (Enzyklopädie der Psychologie, Serie Wirtschafts-, Organisations- und Arbeitspsychologie, Bd. 1., S. 581-622). Göttingen: Hogrefe.
Van den Broeck, A., Vansteenkiste, M., Witte, H., Soenens, B. \& Lens, W. (2010). Capturing autonomy, competence, and relatedness at work: Construction and initial validation of the Workrelated Basic Need Satisfaction scale. Journal of Occupational and Organizational Psychology, 83(4), 981-1002. https://doi. org/10.1348/096317909X481382

Van Vianen, A. E. M. (2018). Person-Environment Fit: A Review of Its Basic Tenets. Annual Review of Organizational Psychology and Organizational Behavior, 5, 75-101. https://doi.org/10. 1146/annurev-orgpsych-032117-104702

Venkatesh, V. \& Davis, F. (2000). A theoretical extension of the technology acceptance model: Four longitudinal field studies. Management Science, 46, $186-204$.

Weiber, R. \& Mühlhaus, D. (2014). Strukturgleichungsmodellierung (Springer-Lehrbuch). Berlin, Heidelberg: Springer. https://doi. org/10.1007/978-3-642-35012-2

\section{Historie}

Eingegangen: 29.05.2021

Revision eingegangen: 24.08. 2021

Onlineveröffentlichung: 22.11.2021

\section{Autorenschaften}

Christian Jaster, jaster@moldzio.com

\section{Förderung}

Open Access-Veröffentlichung ermöglicht durch die Universität Trier.

\section{ORCID}

Christian Jaster

(iD https://orcid.org/0000-0002-1119-3819

Thomas Ellwart

(iD) https://orcid.org/0000-0003-3726-1346

\section{Prof. Dr. Thomas Ellwart}

Fachbereich I - Psychologie

Abteilung Wirtschaftspsychologie

Universität Trier

54286 Trier

ellwart@uni-trier.de 